\title{
Endoscopic removal of recurrent colloid cysts
}

\author{
*Jacques J. Lara-Reyna, MD, ${ }^{1}$ Rafael Uribe-Cardenas, MD, MHS, ${ }^{1}$ Imali Perera, MPH, ${ }^{1}$ \\ Nicholas Szerlip, MD, ${ }^{2}$ Anastasios Giamouriadis, MD, ${ }^{3}$ Nicole Savage, BS, ${ }^{1}$ \\ Therese Haussner, MS, PA-C, ${ }^{1}$ and Mark M. Souweidane, MD1,4
}

\begin{abstract}
1'Department of Neurological Surgery, NewYork-Presbyterian Hospital, Weill Cornell Medical College, New York, New York; 2Department of Neurosurgery, University of Michigan, Ann Arbor, Michigan; ${ }^{3}$ Department of Neuro-Oncology, King's College Hospital, Denmark Hill, London, United Kingdom; and 4Department of Neurosurgery, Memorial Sloan Kettering Cancer Center, New York, New York
\end{abstract}

OBJECTIVE Removal of colloid cysts of the third ventricle using a purely endoscopic method has been established as a safe and advantageous technique. It is hypothesized that endoscopic removal in recurrent cases might pose more technical challenges and result in less success. The objective of this study was to assess the feasibility and outcomes of using a purely endoscopic approach for the management of recurrent colloid cysts compared to primary cysts.

METHODS A retrospective cohort study was performed on patients who underwent purely endoscopic removal of their colloid cyst. Descriptive statistics were compared for patients undergoing surgery for a recurrent cyst and those for a control cohort undergoing surgery for a primary cyst. Bivariate analysis was conducted using a Fisher's exact test for categorical variables and Mann-Whitney U-test for continuous variables.

RESULTS In total, 121 patients had a primary colloid cyst endoscopically removed and 10 patients had a total of 11 recurrent cysts removed. Recurrence or progression after surgery occurred in $3(2.5 \%)$ cases in the primary cyst group and $2(18.2 \%)$ cases in the recurrent cyst group. Symptomatic presentation during the follow-up period occurred in 6 $(54.5 \%)$ cases in the recurrent cyst group versus $75(62 \%)$ cases in the primary cyst group $(p=0.749)$. Two patients $(20 \%)$ in the recurrent group had a second recurrence in a mean period of 30 months (1 patient at 15 and 1 patient at 45 months). One of these patients required a tertiary endoscopic removal 8 years after the second resection. No immediate postoperative complications or new morbidities were observed after repeat endoscopic surgery. The authors' findings indicated a nonsignificant trend toward a higher recurrence rate $(18.2 \% \mathrm{vs} 2.5 \%, p=0.055)$ and a decreased proportion of complete removal $(90.9 \%$ vs $81.8 \%, p=0.296)$ in the recurrent cyst group compared to the primary cyst group. However, a significantly higher rate of preoperative hydrocephalus was observed in the primary cyst group compared with the recurrent cyst group $(63.6 \%$ vs $18.2 \%, p=0.007)$.

CONCLUSIONS Purely endoscopic approaches for the removal of recurrent colloid cysts of the third ventricle are feasible and equally safe compared with endoscopic removal of primary cysts. The study's findings did not show a statistically significant difference in the rate of recurrence between the 2 groups. The proportion of patients with symptomatic cysts on presentation was lower in patients with recurrent cysts than in patients with primary cysts. Due to the high rate of complete removal with negligible morbidity, the authors continue to advocate for an endoscopic removal at the time of cyst recurrence.

https://thejns.org/doi/abs/10.3171/2018.12.JNS181859

KEYWORDS endoscopic; recurrent; colloid cyst; surgical technique

$\mathrm{R}$ ECENT clinical experience has established that the purely endoscopic removal of colloid cysts is a valid and advantageous surgical approach. Benefits associated with endoscopic removal have been reported for length of stay, operating times, and perioperative complications. ${ }^{3,10,12}$ The features and outcomes associated with endoscopic treatment are derived from reports of patients being treated for a newly diagnosed or primary colloid cyst. Whether the same therapeutic benefit of endoscopic surgery can be extrapolated to patients who present with cyst recurrence is unknown. It is hypothesized that the endoscopic removal of recurrent colloid cysts might pose

ABBREVIATIONS EVD = externalized ventricular drain; FOR = frontal occipital horn ratio.

SUBMITTED June 27, 2018. ACCEPTED December 18, 2018.

INCLUDE WHEN CITING Published online April 12, 2019; DOI: 10.3171/2018.12.JNS181859.

${ }^{*}$ J.J.L.R. and R.U.C. contributed equally to this work. 
more challenges and less success due to a distorted anatomy, capsule adherence, indistinct cyst margins, and scar formation. These findings may ultimately translate into higher rates of complications or recurrence. A focused analysis on a cohort of patients undergoing secondary endoscopic removal of a recurrent colloid cyst would provide information to guide current clinical practice. It was our aim to analyze our experience using an endoscopic approach in patients undergoing treatment for recurrent colloid cysts and compare these results with those for patients undergoing treatment for primary colloid cysts.

\section{Methods \\ Clinical Data Collection}

This study was granted an approval by the institutional review board at Weill Cornell Medical College. From a prospective database of patients undergoing endoscopic removal of a colloid cyst, a retrospective review was conducted. The data were collected over a 19-year period (1999-2018) and managed using REDCap (Research Electronic Data Capture). ${ }^{7}$ Patient demographic and clinical information, duration of follow-up, recurrence rate, and surgical complications were considered. Postoperative complications included development of new seizures, intracranial hemorrhage, aseptic meningitis (symptomatic meningitis with a negative CSF culture after 72 hours), bacterial meningitis, wound infection, and ventriculitis (infectious CSF profile after intraventricular surgery).

We identified all patients who had surgery for a recurrent colloid cyst in our patient registry and compared them to a cohort of patients who received treatment for a primary colloid cyst. All patients were treated endoscopically by the same surgeon (M.M.S.). Three patients within the recurrent cyst cohort had been operated on previously by the senior author (M.M.S.).

\section{Endoscopic Technique}

Surgery was performed using a purely endoscopic approach. The procedures were performed with a $0^{\circ}$ and $30^{\circ}, 2-\mathrm{mm}$ rigid lens endoscope through a $6-\mathrm{mm}$ sheath (MINOP, Aesculap). Frameless stereotaxy was used for all procedures for trajectory planning. Cyst removal was accomplished with a combination of cyst aspiration, cyst wall dissection, and bipolar coagulation. The use of an externalized ventricular drain (EVD) was based on intraoperative observation of intraventricular hemorrhage during resection. All procedures were classified at the end of surgery as either complete or incomplete removal based on ventriculoscopic assessment by the senior surgeon (M.M.S.). A complete resection was defined as the removal of the entire intracystic component and capsule attached to the tela choroidea, as confirmed intraoperatively. MRI studies were performed within 48 hours from surgery to establish if any residual lesion was evident, to determine patency of the aqueduct of Sylvius, and to assess ventricular size.

\section{Statistical Analysis}

Descriptive statistics were calculated and bivariate analysis was performed using a Fisher's exact test for categorical variables and Mann-Whitney U-test for continuous variables. Preoperative presence of hydrocephalus was classified by the senior author based on both descriptive and objective (frontal occipital horn ratio [FOR]) measurement data. ${ }^{16}$ An FOR of $>0.37$ was defined as hydrocephalus in this study. This analysis compared cyst recurrence or progression rate, age at surgery, maximal cyst diameter, presence of hydrocephalus, symptomatology, FOR, and complete removal in the primary and recurrent cyst groups. A p value of $<0.05$ was considered statistically significant for this analysis. Statistical analysis was preformed using RStudio.

\section{Results \\ Primary Colloid Cyst Surgery}

This descriptive analysis included 121 patients who underwent primary colloid cyst surgery performed endoscopically (Table 1). This group consisted of 54 males (44.6\%) and 67 females (55.4\%). Forty-six (38\%) patients presented incidentally and $75(62 \%)$ presented as symptomatic. The most common symptoms were headache, nausea or vomiting, visual disturbances, or gait imbalance. Acute decompensation was a presenting symptom in 8 patients, with 6 of these patients requiring preoperative EVD placement. Mean FOR of the primary group was 0.40 (range 0.28-0.52). Sixty-four percent of these patients presented with hydrocephalus, of these $70.1 \%$ were symptomatic.

Average age at surgery was 42.2 years (range 11-73 years). The average cyst diameter was $11.8 \mathrm{~mm}$ (range 3-30 mm). Complete cyst removal was performed in 110 patients $(90.9 \%)$. Thirty-eight patients required placement of an EVD (31.4\%) intraoperatively. Mean length of stay was 2.9 days (range 1-23 days). Postoperative complications included seizure (2/121), aseptic meningitis (2/121), ventriculitis (1/121), titanium burr hole cover dislodgement $(1 / 121)$, and superficial wound infection $(2 / 121)$. Postoperative permanent CSF diversion was required in 5 patients (4 patients with ventriculoperitoneal shunts and 1 with endoscopic third ventriculostomy). The average time of radiological follow-up was 43.7 months (range 1-171 months). Of the 121 patients in the primary group, cyst recurrence or progression of residual cyst occurred in 3 patients $(2.5 \%)$. The average latency period between the first surgery and the diagnosis of the recurrence or progression of residual cyst was 90 months (range 19-132 months).

\section{Recurrent Colloid Cyst Surgery}

A total of 10 patients, 6 women (60\%) and 4 men (40\%), had an endoscopic removal for a cyst recurrence or progression of a residual colloid cyst. This included 3 patients whose previous endoscopic cyst removal was performed by the senior author. Recurrent cysts occurred in 5 patients (50\%) following craniotomy for microsurgical removal at the time of first surgery at outside institutions and in 5 patients (50\%) following endoscopic removal at the time of first surgery. The average latency period from the initial resection to the recurrent surgery was 100 months (range 9-212 months). Two patients (20\%) had an eventual MRdefined subsequent recurrence in a mean time period of 
TABLE 1. Clinical characteristics of patients undergoing endoscopic colloid cyst removal $(n=132)$

\begin{tabular}{|c|c|c|c|}
\hline & Primary Colloid Cyst Op $(n=121)$ & Recurrent Colloid Cyst Op $(n=11)^{*}$ & $\mathrm{p}$ Value \\
\hline Age at op in yrs & $42.2(11-73)$ & $35.1(9-54)$ & 0.170 \\
\hline Symptomatic presentation & $75 / 121(62.0 \%)$ & $6 / 11(54.5 \%)$ & 0.749 \\
\hline Maximal cyst diameter in $\mathrm{mm}$ & $11.8(4-27)$ & $9.8(6-18)$ & 0.138 \\
\hline FOR & $0.40(0.28-0.52)$ & $0.36(0.32-0.41)$ & $0.022 \dagger$ \\
\hline Presence of HCP & $77 / 121(63.6 \%)$ & $2 / 11(18.2 \%)$ & $0.007 \dagger$ \\
\hline Complete removal & $110 / 121(90.9 \%)$ & $9 / 11(81.8 \%)$ & 0.296 \\
\hline Length of stay in days & $2.9(1-23)$ & $2.4(1-10)$ & 0.523 \\
\hline Intraoperative EVD placement & $38 / 121(31.4 \%)$ & $2 / 11(18.2 \%)$ & 0.503 \\
\hline Radiological follow-up in mos & $43.7(1-171)$ & $49(3-143)$ & - \\
\hline Recurrence or progression & $3 / 121(2.5 \%)$ & $2 / 11(18.2 \%)$ & 0.055 \\
\hline Time to recurrence in mos & $90(19-132)$ & $30(15-45)$ & - \\
\hline
\end{tabular}

31 months (15 and 45 months) after the second intervention. One of these patients had progressive cyst growth and underwent a third endoscopic removal 8 years after the second surgery. This resulted in a total of 11 recurrent surgical encounters (10 patients, 1 of whom required 2 surgeries for a recurrence). The mean age at surgery for recurrence was 35.1 years (range 9-54 years). Mean FOR of the recurrent surgery group was 0.36 (range $0.32-0.41$ ). Six recurrences presented as symptomatic (54.5\%). All recurrences were detected by surveillance imaging, with 2 patients presenting with hydrocephalus. The mean maximal cyst diameter at recurrent surgery was $9.8 \mathrm{~mm}$ (range 6-18 $\mathrm{mm}$ ). Nine of the 11 procedures resulted in complete removal at surgery (81.8\%). Two patients required an intraoperative EVD. Mean length of stay was 2.4 days (range 1-10 days). None of the patients in the recurrent group required conversion to a craniotomy. The average radiological follow-up for this group was 49 months (range 3-143 months). No postoperative complications were observed after recurrent surgery. No patient required postoperative CSF diversion.

The clinical characteristics of the recurrent surgical group are presented in Table 2 . There were no statistically significant differences in the recurrence rate, age at surgery, length of stay, clinical presentation, intraoperative EVD use, maximal cyst diameter, and completeness of removal between the 2 groups. We observed statistically significant differences in hydrocephalus $(\mathrm{p}=0.007)$ and FOR $(p=0.022)$ between the primary and recurrent groups.

\section{Case Illustrations}

\section{Patient 2}

A 23-year-old male patient with a history of craniotomy for colloid cyst removal at an outside institution presented with persistent headaches 79 months after surgery. MRI showed recurrence without hydrocephalus (Fig. 1A and B). The relatively short interval from the initial surgery to recurrence, the age of the patient, and the presence of hydrocephalus with clinical symptoms led toward the recommendation of repeat surgery. Endoscopic removal was performed. Intraoperatively, some scar tissue was evident near the foramen of Monro. The cyst capsule was completely removed using endoscopic grasping forceps (Fig. 2). Thirteen months after surgery there had been no recurrence (Fig. $1 \mathrm{C}$ and $\mathrm{D})$.

\section{Patient 5}

A 45-year-old female patient was found to have radiographic progression 10 years after an incomplete initial endoscopic cyst resection. At the time of recurrence she was asymptomatic. However, given the patient's young age and the evident progression in cyst size over time, it was considered necessary to proceed with a second attempt at surgical removal. At the time of secondary surgery, a minimal residual capsule was left behind. Surveillance imaging over the subsequent 8 years demonstrated cyst recurrence and eventual radiographic progression (Fig. 3). A third endoscopic resection was performed with complete removal of the cyst (Fig. 4) confirmed on postoperative MR imaging (Fig. 5).

\section{Discussion}

The endoscopic removal of colloid cysts has been shown to be safe and reliable. ${ }^{914,17,19}$ Advantages of a minimally invasive approach include shorter length of hospital stay, decreased pain, and less surgical morbidity compared to open craniotomy for microsurgical removal. ${ }^{4-6,10-12}$ Recurrent colloid cysts are rare and management strategies vary widely and include microsurgical removal, ventriculoperitoneal or ventriculoatrial shunting, stereotactic aspiration, endoscopic resection, or surveillance imaging. . $^{1,4-6-6,8-13,15 \text {, }}$ ${ }^{18,20}$ Factors such as patient age, the presence of associated hydrocephalus, the size of the cyst, and the presence of associated symptoms must be taken into account when deciding the need for additional interventions.

There is a void in the existing literature regarding the 


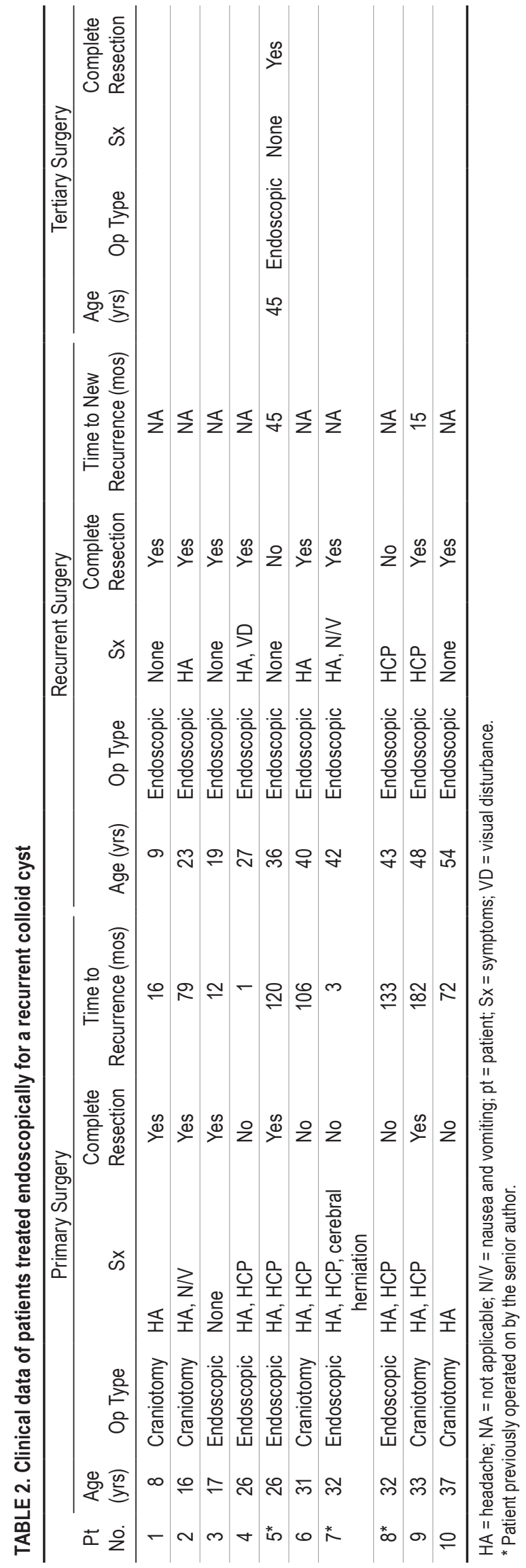

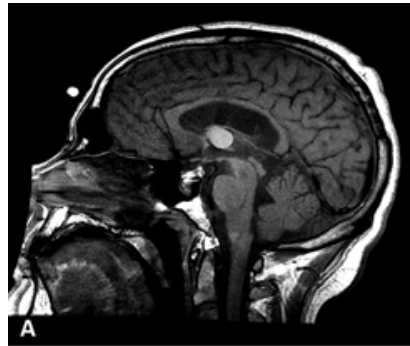
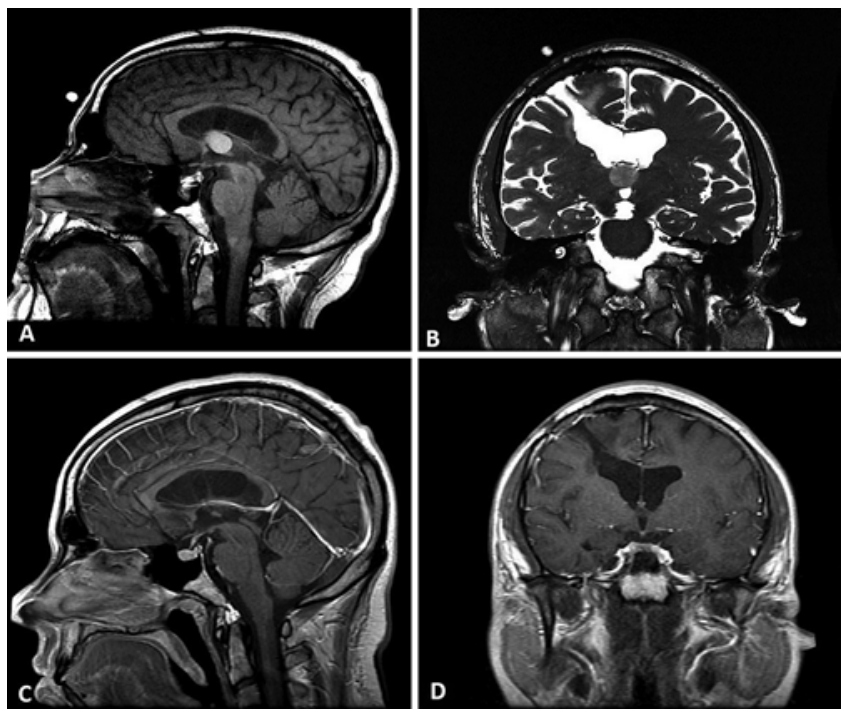

FIG. 1. Preoperative sagittal T1-weighted (A) and coronal T2-weighted (B) MR images displaying a recurrent colloid cyst. Postoperative sagittal (C) and coronal (D) contrast-enhanced T1-weighted MR images after complete secondary endoscopic removal of the recurrent cyst.

optimal management scheme for patients with recurrent colloid cysts. We did not find studies that exclusively addressed patients with recurrent cysts or comparison studies between primary and recurrent endoscopic surgical treatment. Reasonably if the same benefits can be assigned to patients at recurrence then an endoscopic alternative remains a legitimate option for secondary surgery.

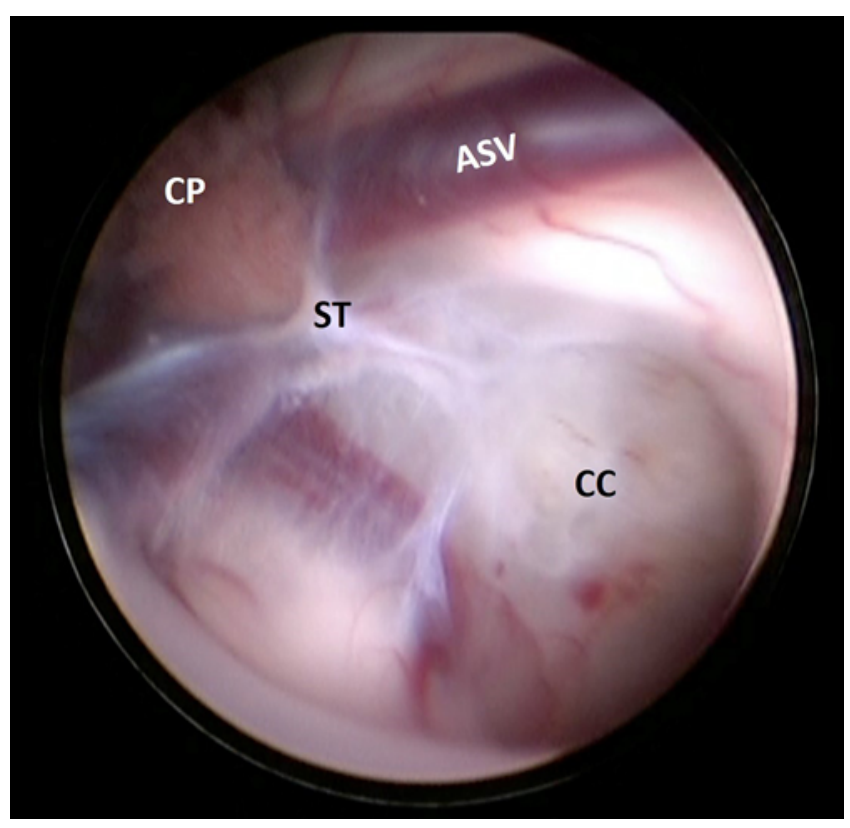

FIG. 2. Right ventricular endoscopic view demonstrating the typical synechiae around a recurrent colloid cyst and choroidal fissure. ASV = anterior septal vein; $\mathrm{CC}=$ colloid cyst; $\mathrm{CP}$ = choroid plexus; $\mathrm{ST}$ = scar tissue. Figure is available in color online only. 


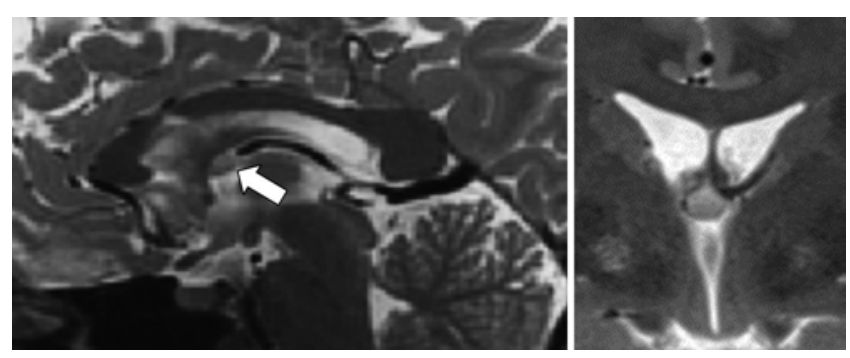

FIG. 3. Sagittal (left) and coronal (right) T2-weighted MR images displaying recurrent colloid cyst (white arrow) before tertiary endoscopic resection.

Indications for treatment in our sample of recurrent cases were based on radiographic progression of cyst size, increasing ventricular enlargement, symptomatology, and age. ${ }^{14}$ In our series, 6 patients in the recurrent cyst group presented with symptoms of progression or recurrence during the postsurgery follow-up period $(54.5 \%)$ versus 75 patients $(62 \%)$ in the primary group. The difference in the proportion of symptomatic patients between groups was not statistically significant $(p=0.749)$. Similarly, the difference between groups in age and maximal cyst diameter was not statistically significant. However, we did observe a significant difference in preoperative hydrocephalus (63.6\% vs $18.2 \%, \mathrm{p}=0.007)$ and FOR (0.40 vs $0.36, \mathrm{p}$ $=0.022$ ) between the primary and recurrent groups. Although our sample was not large enough to detect a statistically significant difference in the proportion of symptomatic patients between both groups, there was a trend for patients with recurrent cysts to be less symptomatic than those in the primary group. The lower rates of hydrocephalus and the smaller proportion of symptomatic patients in the recurrent group are probably explained by the fact that patients are followed closely after initial resection, allowing for an earlier diagnosis of recurrence before hydrocephalus and elevated intracranial pressure develop again.

Our results showed a higher rate of complete resection (90.9\% vs $81.8 \%, \mathrm{p}=0.296$ ) and lower rate of recurrence $(3.3 \%$ vs $18.2 \%, p=0.055)$ in the primary group compared to the recurrent group. These findings were not statistically significant, a result that is most likely related to sample
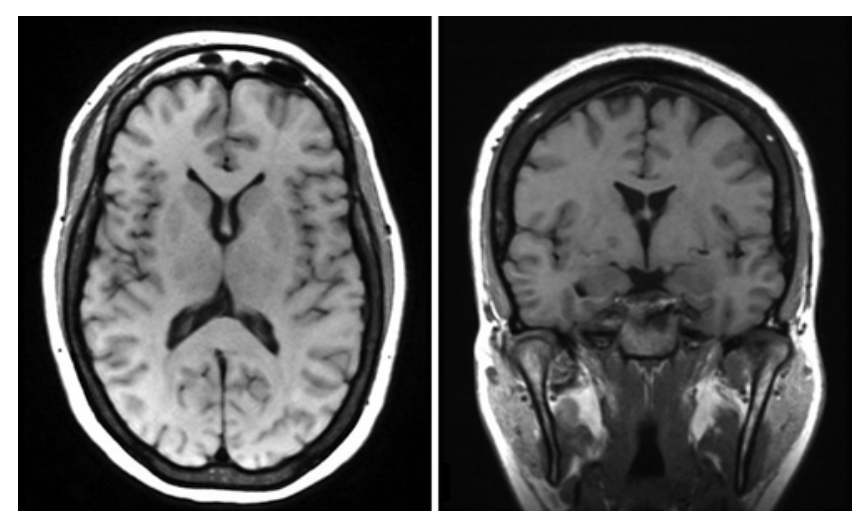

FIG. 5. Postoperative T1-weighted MRI in axial (left) and coronal (right) views showing no remnant or residual cyst.

size. This trend is not unexpected if one assumes that scarring around the foramen of Monro might preclude complete removal of the cyst capsule at the time of revision surgery. Theoretically, that concept would apply similarly for cases in which patients undergo microsurgical resection and should not be used as a reason to favor one form of treatment over another.

We found lower rates of EVD placement in the recurrent group (18.2\%) compared to the primary group (31.4\%), although this trend was not significant. The significance of these findings is limited by sample size; however, it is most likely a reflection of the evolution of the technique and an overall trend for less use of drains at the end of surgery.

There were no surgical complications reported in any of the recurrent cases. The 2 patients in the recurrent group who required an EVD did not go on to require additional CSF diversion. Our findings suggest that the endoscopic approach is a valid alternative for the treatment of patients with recurrent colloid cysts. These findings, however, must be taken with caution. The retrospective nature of the data collected raises the potential for selection bias within this study. Furthermore, our findings represent a singlesurgeon, single-center experience and therefore might not be generalizable to hospitals where these procedures are not routinely performed. Finally, it is possible that some recurrences from the registry of primary surgeries were not diagnosed due to loss to follow-up.
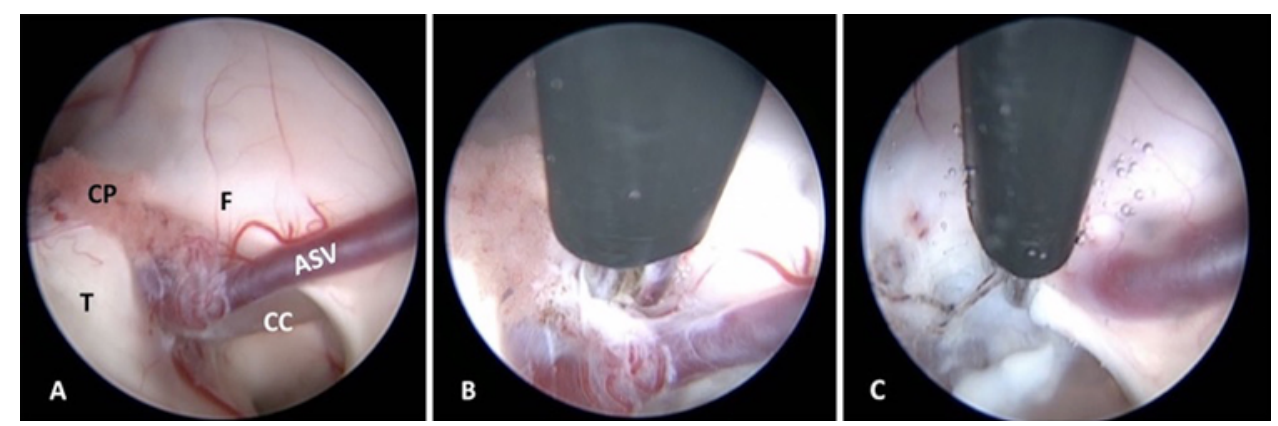

FIG. 4. A right transforaminal view demonstrating limited exposure of the colloid cyst $(\mathbf{A})$. A transchoroidal approach was utilized (B) by transecting the anterior septal vein to better visualize the colloid cyst $(\mathrm{C})$. $\mathrm{ASV}=$ anterior septal vein; $\mathrm{CC}=\mathrm{colloid}$ cyst; $\mathrm{CP}=$ choroid plexus; $F=$ fornix; $T$ = thalamus. Figure is available in color online only. 


\section{Conclusions}

The optimal management scheme of patients with recurrent colloid cysts is unknown. Young age, the presence of hydrocephalus, large cyst size, and the presence of symptoms are the main indications to proceed with surgery. In experienced centers, endoscopic approaches afford the possibility of a shorter length of stay, decreased surgical morbidity, and comparable rates of complete resection compared to primary cases. Comparable studies of recurrent cysts treated via microsurgical removal are lacking and small sample sizes limit the generalizability of results to the wider population. Multicenter studies designed to create larger patient registries are needed in order to better understand the natural history of recurrent colloid cysts and the optimal form of management.

\section{References}

1. Birski M, Birska J, Paczkowski D, Furtak J, Rusinek M, Rudas M, et al: Combination of neuroendoscopic and stereotactic procedures for total resection of colloid cysts with favorable neurological and cognitive outcomes. World Neurosurg 85:205-214, 2016

2. Boogaarts HD, Decq P, Grotenhuis JA, Le Guérinel C, Nseir $\mathrm{R}$, Jarraya B, et al: Long-term results of the neuroendoscopic management of colloid cysts of the third ventricle: a series of 90 cases. Neurosurgery 68:179-187, 2011

3. Chibbaro S, Champeaux C, Poczos P, Cardarelli M, Di Rocco $\mathrm{F}$, Iaccarino C, et al: Anterior trans-frontal endoscopic management of colloid cyst: an effective, safe, and elegant way of treatment. Case series and technical note from a multicenter prospective study. Neurosurg Rev 37:235-241, 2014

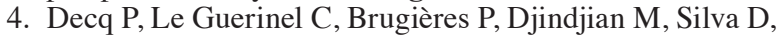
Kéravel Y, et al: Endoscopic management of colloid cysts. Neurosurgery 42:1288-1296, 1998

5. Greenlee JD, Teo C, Ghahreman A, Kwok B: Purely endoscopic resection of colloid cysts. Neurosurgery 62 (3 Suppl 1):51-56, 2008

6. Grondin RT, Hader W, MacRae ME, Hamilton MG: Endoscopic versus microsurgical resection of third ventricle colloid cysts. Can J Neurol Sci 34:197-207, 2007

7. Harris PA, Taylor R, Thielke R, Payne J, Gonzalez N, Conde JG: Research electronic data capture (REDCap) - a metadata-driven methodology and workflow process for providing translational research informatics support. J Biomed Inform 42:377-381, 2009

8. Hellwig D, Bauer BL, Schulte M, Gatscher S, Riegel T, Bertalanffy H: Neuroendoscopic treatment for colloid cysts of the third ventricle: the experience of a decade. Neurosurgery 52:525-533, 2003

9. Hoffman CE, Savage NJ, Souweidane MM: The significance of cyst remnants after endoscopic colloid cyst resection: a retrospective clinical case series. Neurosurgery 73:233-239, 2013

10. Horn EM, Feiz-Erfan I, Bristol RE, Lekovic GP, Goslar PW, Smith KA, et al: Treatment options for third ventricular colloid cysts: comparison of open microsurgical versus endoscopic resection. Neurosurgery 60:613-620, 2007
11. Kehler U, Brunori A, Gliemroth J, Nowak G, Delitala A, Chiappetta F, et al: Twenty colloid cysts-comparison of endoscopic and microsurgical management. Minim Invasive Neurosurg 44:121-127, 2001

12. Levine NB, Miller MN, Crone KR: Endoscopic resection of colloid cysts: indications, technique, and results during a 13 year period. Minim Invasive Neurosurg 50:313-317, 2007

13. Longatti P, Godano U, Gangemi M, Delitala A, Morace E, Genitori L, et al: Cooperative study by the Italian neuroendoscopy group on the treatment of 61 colloid cysts. Childs Nerv Syst 22:1263-1267, 2006

14. Margetis K, Christos PJ, Souweidane M: Endoscopic resection of incidental colloid cysts. J Neurosurg 120:1259-1267, 2014

15. Mishra S, Chandra PS, Suri A, Rajender K, Sharma BS, Mahapatra AK: Endoscopic management of third ventricular colloid cysts: eight years' institutional experience and description of a new technique. Neurol India 58:412-417, 2010

16. O'Hayon BB, Drake JM, Ossip MG, Tuli S, Clarke M: Frontal and occipital horn ratio: a linear estimate of ventricular size for multiple imaging modalities in pediatric hydrocephalus. Pediatr Neurosurg 29:245-249, 1998

17. Qiao L, Souweidane MM: Purely endoscopic removal of intraventricular brain tumors: a consensus opinion and update. Minim Invasive Neurosurg 54:149-154, 2011

18. Schroeder HW, Gaab MR: Endoscopic resection of colloid cysts. Neurosurgery 51:1441-1445, 2002

19. Souweidane MM: Endoscopic surgery for intraventricular brain tumors in patients without hydrocephalus. Neurosurgery 57 (4 Suppl):312-318, 2005

20. Sribnick EA, Dadashev VY, Miller BA, Hawkins S, Hadjipanayis CG: Neuroendoscopic colloid cyst resection: a case cohort with follow-up and patient satisfaction. World Neurosurg 81:584-593, 2014

\section{Disclosures}

Mark M. Souweidane is a paid consultant for Aesculap, a division of B. Braun Melsungen AG.

\section{Author Contributions}

Conception and design: Souweidane, Szerlip, Giamouriadis, Savage. Acquisition of data: Souweidane, Perera, Szerlip, Giamouriadis, Savage, Haussner. Analysis and interpretation of data: LaraReyna, Uribe-Cardenas, Perera. Drafting the article: all authors. Critically revising the article: all authors. Reviewed submitted version of manuscript: all authors. Statistical analysis: LaraReyna, Uribe-Cardenas, Perera. Administrative/technical/material support: Souweidane, Lara-Reyna, Uribe-Cardenas, Perera, Haussner. Study supervision: Souweidane.

\section{Correspondence}

Mark M. Souweidane: NewYork-Presbyterian Hospital, Weill Cornell Medical College, New York, NY. mmsouwei@med. cornell.edu. 